\title{
Time-varying Stiffness Characteristics of Shaft with Slant Crack
}

\author{
Hengheng Xia \\ School of Aeronautical Manufacturing Engineering \\ Nanchang Hangkong University \\ Nanchang, China
}

\author{
Yaoxian Xiao \\ School of Aeronautical Manufacturing Engineering \\ Nanchang Hangkong University \\ Nanchang, China
}

\author{
Zhinong $\mathrm{Li} *$ \\ School of Aeronautical Manufacturing Engineering \\ Nanchang Hangkong University \\ Nanchang, China
}

\begin{abstract}
The stiffness matrix of a shaft with slant transverse crack under the common action of axial force, moment and torque was derived based on material mechanics, local flexibility and fracture mechanics theory and apply strain energy release rate method. The opening and closing regularity of the crack and the shaft rigidity varying characteristics was simulated in a steady state rotating by apply the stress intensity factor is zero method, and the time-varying characteristics of inclination of $45^{\circ}$ cracked shaft stiffness was detailed study. The studies have shown that, the stiffness of shaft has not been reduced, but has some breathing characteristics of fluctuations with increasing crack depth in the steady state crack axis of rotation cycle. And the coupled vibration was easily caused so that dynamic characteristics of shaft is more complex.
\end{abstract}

Keywords-slant-cracked shaft; shaft stiffness; time-variant characteristics

\section{INTRODUCTION}

Nonlinear dynamic characteristics of a cracked shaft is mainly affected by the time-varying stiffness of the shaft, us establishing reasonable model for calculating crack shaft stiffness is one of the important content to study the dynamic characteristics of the shaft crack. In fact, since many of the rotor systems are used to transmit torque, so slant crack produce inevitable. And how to accurately and precisely describe the rigidity of slant crack rotor system is the key to studying the characteristics of slant crack rotor system dynamics and the foundation.

The study of slant crack rotor system began in twentieth Century 80 time, and have made some progress [1-9]. Lin Yanli [5-8] did the research based on stress intensity factor is zero method and made out the influencing of load and crack depth on shaft stiffness .Darpe[9] analysis additional flexibility and stiffness matrix of arbitrary angle crack rotor under the effect of 6 kinds of loads, but he did not work out the influence of crack opening and closing of a shaft stiffness when the rotor is rotate.However, the literature [1-8] did not consider the open-close regularity of crack and the time-varying characteristics of rotor stiffness in a steady rotation cycle. Obviously, the change rule of crack shaft stiffness has the very big difference in the stationary state and rotating condition. Though the literature [9] has been considered the crack opening and closing law and time varying stiffness characteristics in steady rotation cycle, however, this is only a diagnosis of slant crack rotor of 45, and ignore the influence of crack angle of slant crack rigidity, has obvious shortage.

This paper derivates stiffness matrix by the way of strain energy release rates which was proposed by Dimarogonas, using the factor that Stress intensity factor is zero,which was proposed by Darpe to simulate the crack breathing properties, and on this basis, study the crack opening and closing law and time varying stiffness characteristics of the $45^{\circ}$ slant crack shaft in steady rotation cycle.

\section{Slant CRACK MOdEL OF THE ROTOR SYSTEM}

Consider a shaft of radius $\mathrm{R}$ with a slant crack having a depth of $\alpha$ oriented at angle of $\beta$ relative to the axis of the shaft (Fig.1(a)).Fig.1(b) shows the details of the cross-section showing open and closed area to the crack above the crack edge ,the shaft is loaded with axial force $p_{1}$, bending moment $p_{2}$ and $p_{3}$ and torque $p_{4}$.

The opening mode stress intensity factors are given as below:

$$
\begin{gathered}
P_{1}: K_{\mathrm{I} 1}=\frac{P_{1}}{2 \pi R^{2}}(1+\cos 2 \beta) \sqrt{\pi a} F_{1} \\
K_{\mathrm{III} 1}=\frac{P_{1}}{2 \pi R^{2}} \sin 2 \beta \sqrt{\pi a} F_{\mathrm{III}} \\
P_{2}: K_{\mathrm{I} 2}=\frac{2 P_{2} Y}{\pi R^{4}}(1+\cos 2 \beta) \sqrt{\pi a} F_{1}
\end{gathered}
$$




$$
\begin{gathered}
P_{3}: K_{\mathrm{I} 3}=\frac{P_{3} h}{\pi R^{4}}(1+\cos 2 \beta) \sqrt{\pi a} F_{2} \\
K_{\mathrm{III} 3}=\frac{P_{3} h}{\pi R^{4}} \sin (2 \beta) \sqrt{\pi a} F_{\mathrm{III}} \\
P_{4}: K_{\mathrm{I} 4}=\frac{2 P_{4} \sqrt{Y^{2}+\left(\frac{h}{2}\right)^{2}}}{\pi R^{4}} \sin (2 \beta) \sqrt{\pi a} F_{2}
\end{gathered}
$$

Therefore, the total stress intensity factor is given by following expression:

$$
\begin{gathered}
K_{\mathrm{I}}=K_{\mathrm{I} 1}+K_{\mathrm{I} 2}+K_{\mathrm{I} 3}+K_{\mathrm{I} 4} \\
K_{\mathrm{III}}=K_{\mathrm{III} 1}+K_{\mathrm{III} 3}
\end{gathered}
$$

Among them, assuming $\lambda=\frac{\pi a}{2 h}$, according to the literature [10], three influence coefficients is given by following expression:

$$
\begin{gathered}
F_{1}=\sqrt{\frac{\tan \lambda}{\lambda}}\left(0.752+2.02(\alpha / h)+0.37(1-\sin \lambda)^{3}\right) \frac{1}{\cos \lambda} \\
F_{2}=\sqrt{\frac{\tan \lambda}{\lambda}}\left[0.923+0.199(1-\sin \lambda)^{4}\right] \frac{1}{\cos \lambda} \\
F_{\mathrm{III}}=\sqrt{\frac{\tan \lambda}{\lambda}}
\end{gathered}
$$

Where $J$ is strain energy density function and is expressed as:

$$
J=\frac{1}{E^{\prime}}\left[K_{\mathrm{I}}^{2}+(1+v) K_{\mathrm{III}}^{2}\right]
$$

Suppose $A^{\prime}$ for crack opening area (The first stress intensity factor $K_{\mathrm{I}}>0$ ), the crack rotor release of strain energy can be expressed as:

$$
W=\iint_{A^{\prime}} \frac{1}{E^{\prime}} K_{\mathrm{I}}^{2} d \alpha d Y+\iint_{A^{\prime}} \frac{1+v}{E^{\prime}} K_{\mathrm{III}}^{2} d a d Y
$$

Using Castigliano theorem, the additional flexibility can be expressed as:

$$
\Delta c_{i j}=\frac{\partial^{2} W}{\partial q_{i} \partial q_{j}}
$$

Where $\boldsymbol{c}_{s}$ is no crack shaft flexibility and is expressed as:

$$
c_{s}=\operatorname{diag}\left[\begin{array}{llll}
\frac{l^{3}}{48 E I} & \frac{l^{3}}{48 E I} & \frac{l}{2 E A} & \frac{l}{2 E A}
\end{array}\right]
$$

Flexibility matrix can be expressed as follows:

$c_{11}=\frac{1}{E^{\prime} \pi R^{4}} \iint\left[2 \alpha \sin ^{4} \beta F_{\mathrm{I}}+2(1+v) \alpha \sin ^{2} \beta \cos ^{2} \beta F^{2}{ }_{\mathrm{III}}\right] d A^{\prime}$

$c_{22}=\frac{1}{E^{\prime} \pi R^{8}} \iint\left[32 y^{2} \alpha \sin ^{4} \beta F^{2} I+32 y^{2}(1+v) \alpha \sin ^{2} \beta \cos ^{2} \beta F^{2}{ }_{I I I}\right]$

$c_{33}=\frac{1}{E^{\prime} \pi R^{8}} \iint\left[8 \boldsymbol{h}^{2} \alpha \sin ^{4} \beta F^{2}{ }_{2}+8(1+v) h^{2} \alpha \sin ^{2} \beta \cos ^{2} \beta F^{2}{ }_{\text {III }}\right] d A^{\prime}$

$c_{44}=\frac{1}{E^{\prime} \pi R^{8}} \iint\left[2 h^{2} \alpha \sin ^{2} 2 \beta F^{2}{ }_{2}+8 y^{2} \alpha \sin ^{2} \beta \cos ^{2} \beta F^{2}{ }^{2}+2 \alpha(1+v) \cos ^{2} 2 \beta F_{\text {III }}\right] d A^{\prime}$

$c_{12}=\frac{1}{E^{\prime}} \iint\left[\frac{8 y \alpha \sin ^{4} \beta F_{\mathrm{I}}^{2}}{\pi R^{6}}+8(1+v) \frac{y \sin ^{2} \beta \cos ^{2} \beta \alpha F^{2}{ }_{\mathrm{III}}}{\pi R^{6}}\right] d A^{\prime}$

$c_{13}=\frac{1}{E^{\prime}} \iint\left[\frac{2 \boldsymbol{h} \sin ^{4} \beta \alpha F_{\mathrm{I}} F_{2}}{\pi \boldsymbol{R}^{6}}+2(1+v) \frac{h \sin ^{2} \beta \cos ^{2} \beta \alpha \mathrm{F}^{2}{ }_{\mathrm{III}}}{\pi R^{6}}\right] d A^{\prime}$

$c_{14}=\frac{1}{E^{\prime}} \iint\left[\frac{h \sin ^{2} \beta \sin 2 \beta \alpha F_{1} F_{2}}{\pi R^{6}}+2(1+v) \frac{h \sin \beta \cos \beta \cos 2 \beta \alpha F^{2} \text { III }}{\pi R^{6}}\right] d A^{\prime}$

$c_{23}=\frac{1}{E^{\prime}} \times \iint\left[\frac{8 y \sin ^{4} \beta \alpha h F_{1} F_{2}}{\pi R^{8}}+8(1+v) \frac{y h \sin ^{2} \beta \cos ^{2} \beta \alpha F^{2}{ }_{\text {III }}}{\pi R^{8}}\right] d A^{\prime}$

$c_{24}=\frac{1}{E^{\prime}} \iint\left[\frac{4 y \sin ^{2} \beta \alpha \sin 2 \beta F_{1} F_{2}}{\pi R^{8}}+4(1+v) \frac{h y \alpha \sin \beta \cos \beta \cos 2 \beta F^{2} \text { III }}{\pi R^{8}}\right] d A^{\prime}$

$c_{34}=\frac{1}{E^{\prime}} \iint\left[\frac{16 \sin ^{2} \beta \alpha \sin 2 \beta h^{2} F^{2} 2}{\pi R^{8}}+16(1+v) \frac{h^{2} \alpha \sin \beta \cos 2 \beta \cos \beta F^{2} \mathrm{III}}{\pi R^{8}}\right] d A^{\prime}$

Therefore, crack shaft flexibility matrix as follows:

$$
\boldsymbol{c}=\left(\begin{array}{llll}
\boldsymbol{c}_{11} & c_{12} & c_{13} & c_{14} \\
c_{21} & c_{22} & c_{23} & c_{24} \\
c_{31} & c_{32} & c_{33} & c_{34} \\
c_{41} & c_{42} & c_{43} & c_{44}
\end{array}\right)
$$

The stiffness matrix for inverse matrix flexibility matrix: 


$$
\boldsymbol{k}=\left(\begin{array}{llll}
\boldsymbol{k}_{11} & k_{12} & k_{13} & k_{14} \\
k_{21} & k_{22} & k_{23} & k_{24} \\
k_{31} & k_{32} & k_{33} & k_{34} \\
k_{41} & k_{42} & k_{43} & k_{44}
\end{array}\right)=\left(\begin{array}{llll}
c_{11} & c_{12} & c_{13} & c_{14} \\
c_{21} & c_{22} & c_{23} & c_{24} \\
c_{31} & c_{32} & c_{33} & c_{34} \\
c_{41} & c_{42} & c_{43} & c_{44}
\end{array}\right)
$$

By no crack shaft flexibility of $\boldsymbol{c}_{s}$ can get no crack shaft stiffness matrix $\boldsymbol{k}_{\boldsymbol{s}}$, Then the matrix $\overline{\boldsymbol{k}}$ can be obtained by dividing the $\boldsymbol{k}_{\mathrm{s}}$ matrix with $\boldsymbol{k}$ which is expressed as:

$$
\begin{gathered}
\boldsymbol{k}_{s}=\operatorname{diag}^{-1}\left[\begin{array}{cccc}
\frac{l^{3}}{48 E I} & \frac{l^{3}}{48 E I} & \frac{l}{2 E A} & \frac{l}{2 E A}
\end{array}\right] \\
\overline{\boldsymbol{k}}=\boldsymbol{k}_{\boldsymbol{s}}^{-1} \boldsymbol{k}
\end{gathered}
$$

\section{OPENING AND CLOSING CHARACTERISTICS OF SLANT CRACK SECTION AND STIFFNESS ANALYSIS}

Figure 2 shows that, when the crack depth is shallow, the cracked shaft stiffness variation of the main direction is not obvious, with the increase of crack depth, the stiffness have a certain decline, when the crack depth is deeper, the axial direction crack stiffness decrease maximum, axial and torsion direction stiffness changes are symmetrical about angle 180, while the two bending stiffness is not symmetrical about angle 180 , and two bending stiffness variation range is about the same, crack torsion direction stiffness change trend is more close to the axial stiffness; the stiffness change of axial ,torsion and transverse bending direction were decreased first and then increased, while the stiffness $\left(\mathrm{k}_{33}\right)$ of bending direction with the above three direction stiffness change trend is different, it is first decreased and then have a modest rise then hit bottom, finally returned to the origin of the trend.

Due to the rotation of the rotor will generate a certain degree of vibration, which produces the coupling stiffness; it is concluded that the bending coupling stiffness $\left(k_{23}\right)$ and the coupling stiffness $\left(k_{34}\right)$ is almost zero through calculation and analysis, so did not give the two direction stiffness variation map; can be seen from the graph, , the coupling stiffness change is not obvious when the crack depth is shallow, with the increase of crack depth, the changes gradually become obvious; axial and bending direction of the coupling stiffness $\left(k_{12}\right)$ as the rotation of the rotor angle change no longer has been positive, but fluctuate around zero, similar to sine curve changes, axial stiffness and bending stiffness coupling $\left(k_{13}\right)$ between angle of $0^{\circ}$ and $280^{\circ}$ trend similar to the main direction of $k_{11}$ and $k_{44}$ change, but in the $280^{\circ}$ to $360^{\circ}$ is a small bump. the coupled stiffness $\left(k_{\mathbf{1 4}}\right)$ changes trend of axial and torsion direction are similar to the main direction of $k_{11}$ and $k_{44}$ with the change of angle in a complete cycle, just the value for the negative, coupling stiffness of bending and torsion direction $\left(k_{24}\right)$ along with the rotation Angle changes are similar to the sine curve, and like the coupling stiffness $\left(k_{12}\right)$, but the change range is smaller; Can also see that the coupling stiffness of all the direction are zero when the rotation of the rotor angle is 0 degree in the initial moments, the above analysis can know that more coupling vibration will make crack rotor dynamic performance more complicated.

\section{CONCLUSION}

This paper is analyzed from the time-varying characteristics of slant crack:

The cracked shaft stiffness of the main direction along with crack angle is not obvious under the crack depth is shallow when the crack shaft to rotate, the stiffness of the main direction have a degree of decline with the increase of crack depth, the stiffness of axial crack direction decline the largest when the crack depth is deeper; the stiffness change in the direction of axial and transverse bending and torsion were decreased first and then increased; for coupling stiffness, the stiffness change is not obvious when the crack depth is shallow, but the changes gradually become obvious with the increase of crack depth, the coupled stiffness $\left(k_{12}\right)$ change of axial and bending direction is similar to sine curve with shaft angle changes, the coupled stiffness $\left(k_{24}\right)$ change of bending and torsion direction is similar to sine curve with shaft angle changes, all the coupling direction stiffness at the initial moment stiffness is zero.

\section{ACKNOWLEDGEMENT}

This work was financially supported by the Natural Science Foundation of China (51265039, 51261024, 51075372, 50775208) and the Graduate Innovation Found of Jiangxi Province (YC2014-S389).

\section{REFERENCE}

[1] Ichimonji $\mathrm{M}$ and Watanabe $\mathrm{S}$. The dynamics of a rotor system with a shaft having a slant crack. JSME, International Journal, Series III 1988 ,31(4), 712-718.

[2] Ichimonji M, Kazao Y, Watanabe S and Nonaka S. The dynamics of a rotor system with a slant crack under torsional vibration. Nonlinear and Stochastic Dynamics, 1994,78: 81-89.

[3] Prabhakar S, Sekhar A S and Mohanty A R. Transient analysis of a slant-cracked rotor passing through its flexural the critical speed. Mechanism and Machine Theory, 2002,37(9):1007-1020.

[4] Sekhar A S, Mohanty A R and Prabhakar S. Vibrations of cracked rotor system: transverse crack versus slant crack. Journal of Sound and Vibration, 2005,279:1203-1217.

[5] Lin Y, Chu F. The dynamic behavior of a rotor system with a slant crack on the shaft[J]. Mechanical Systems and Signal Processing, 2010, 24(2): 522-545.

[6] Yanli Lin, Fulei Chu, Numerical and experimental investigations of flexural vibrations of a rotor system with transverse or slant crack, Journal of Sound and Vibration, Vol.324, Nos.1-2, 10 July 2009.

[7] Lin Yanli. Dynamic characteristics of the rotor system with a slant crack on the shaft[D].Beijing: Tsinghua University,2008.

[8] Lin Yanli, Chu Fulei, Hao Rujiang, etc. Dynamic Analysis of a Jeffcott Rotor System with an Opening Slant Crack on Its Shaft[J]. Journal of Vibration and Shock, 2008, 27(1): 25-29.

[9] Darpe A K. Dynamics of a Jeffcott rotor with slant crack[J]. Journal of Sound and Vibration, 2007, 303(1): 1-28. 


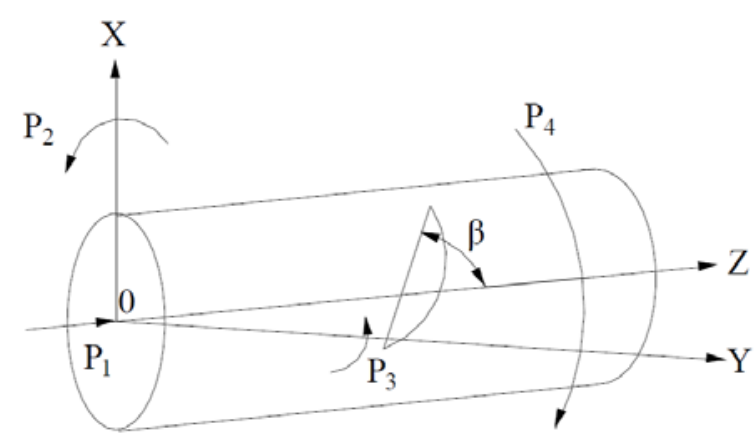

(a)

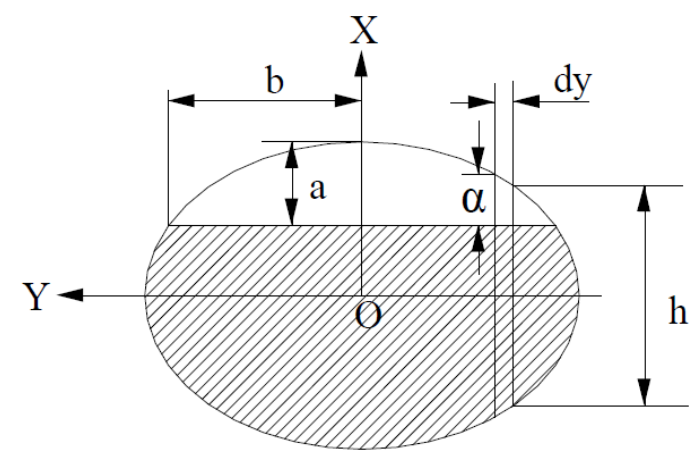

(b)

FIGURE I SLANT CRACK SHAFT AND SLANT CRACK SECTION

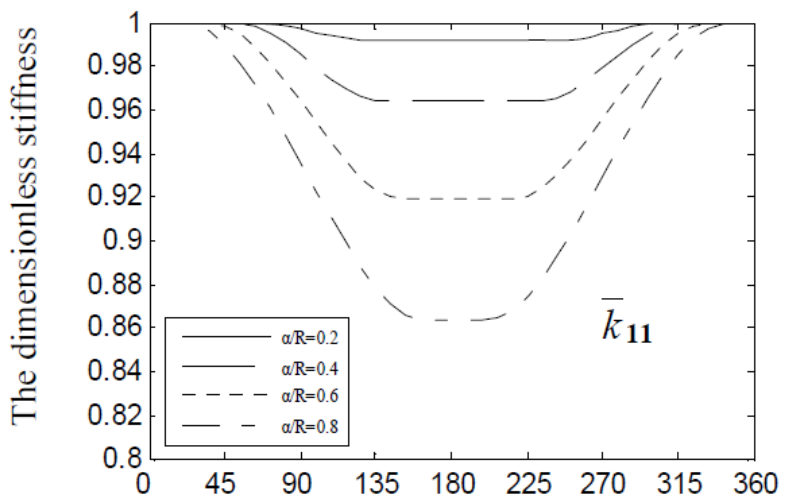

(a) Angle of rotation

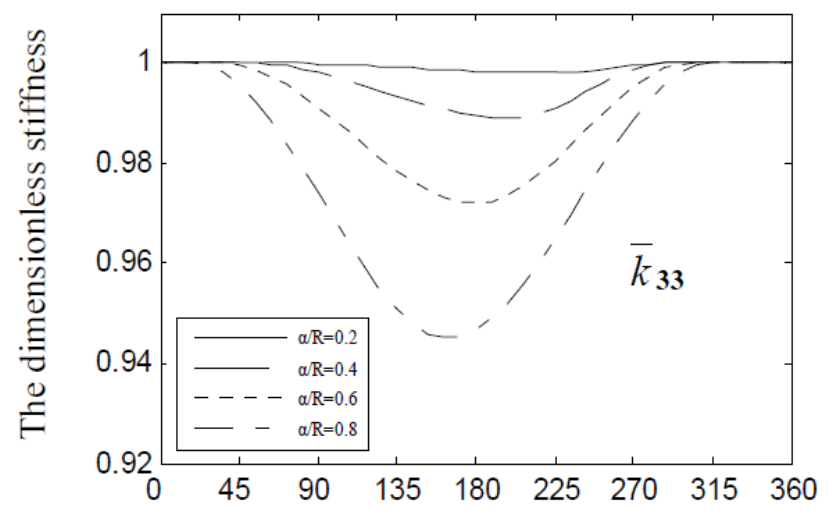

(c) Angle of rotation

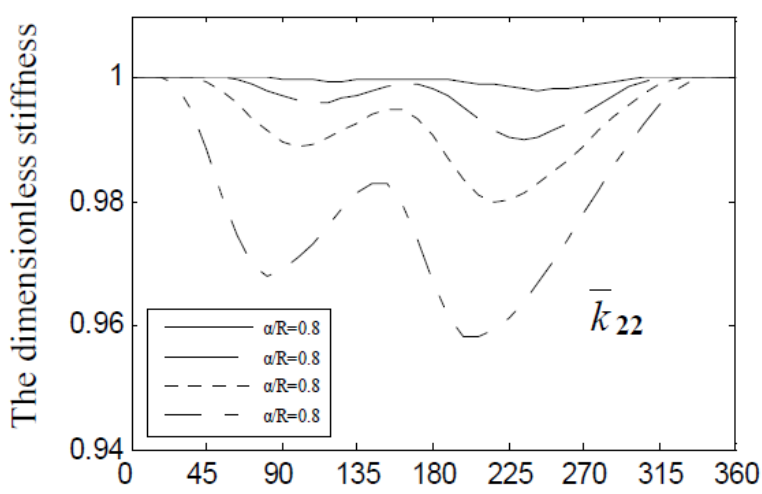

(b) Angle of rotation

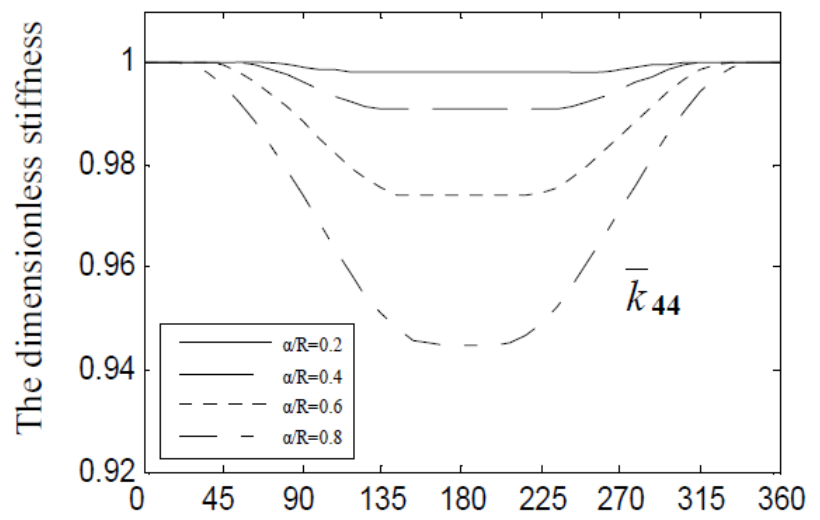

(d) Angle of rotation 


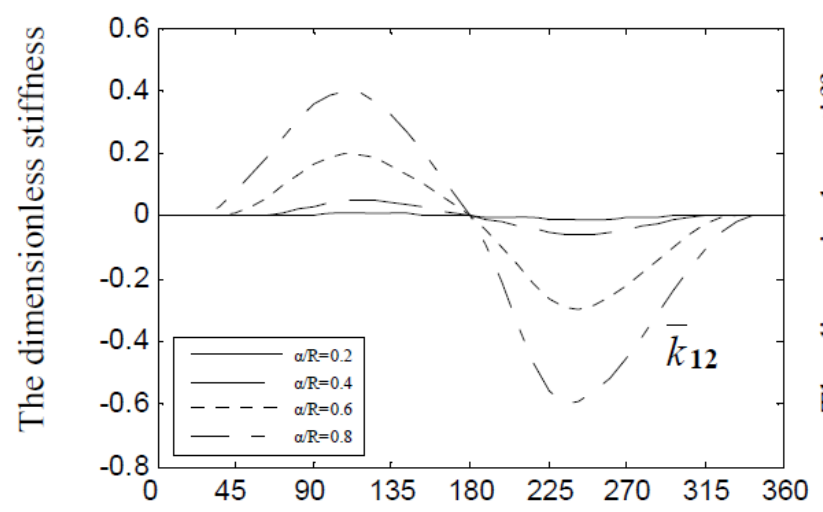

(e) Angle of rotation

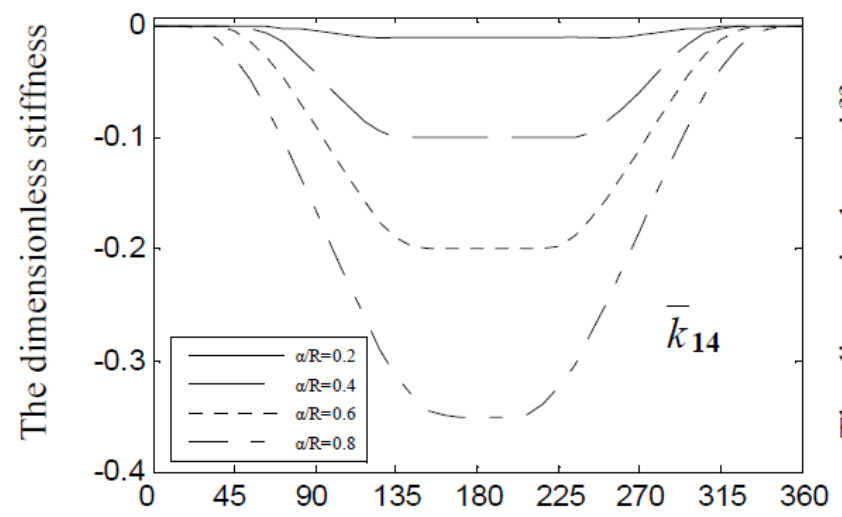

(g) Angle of rotation $\quad(\mathrm{h})$ Angle of rotation

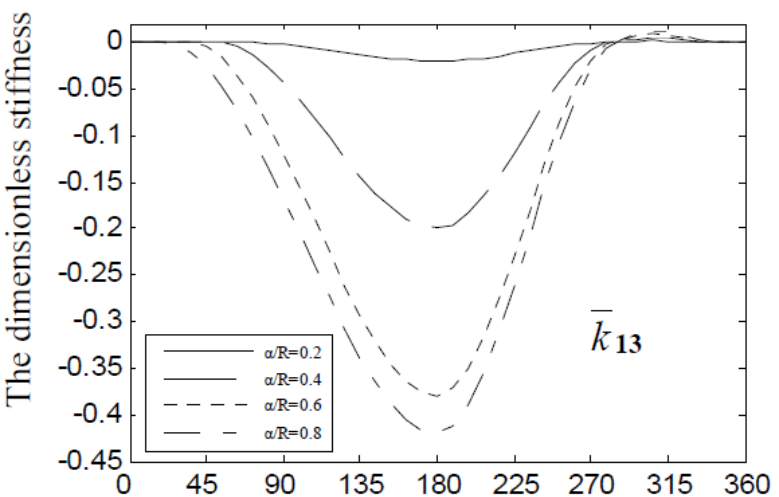

(f) Angle of rotation

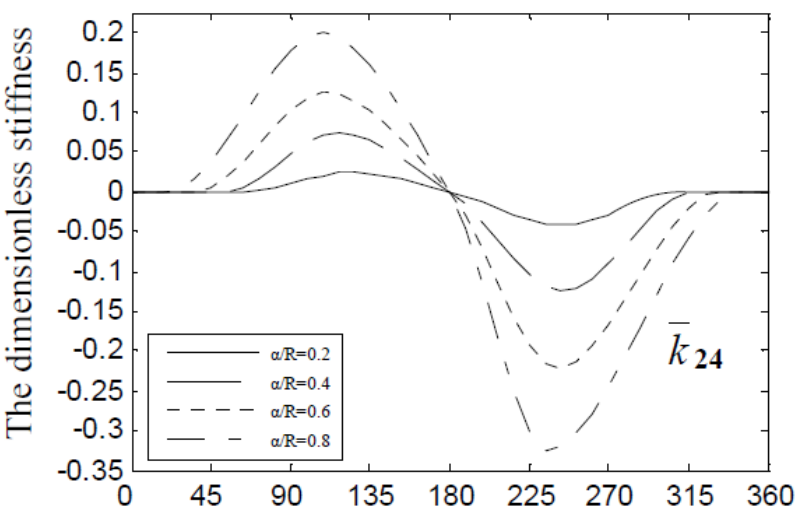

FIGURE II CRACK SHAFT STIFFNESS CHANGES WITH THE ROTATION ANGLE AND THE DEPTH OF THE CRACK 